\title{
Metabolic Effects of Bovine Milk Oligosaccharides on Selected Commensals of the Infant Microbiome- Commensalism and Postbiotic Effects
}

\author{
Louise M. A. Jakobsen ${ }^{1,2, *} \mathbb{C}$, Maria X. Maldonado-Gómez ${ }^{2}$, Ulrik K. Sundekilde ${ }^{1}(\mathbb{D}$, \\ Henrik J. Andersen ${ }^{3}$, Dennis S. Nielsen ${ }^{4}$ and Hanne C. Bertram ${ }^{1}$ (D) \\ 1 Department of Food Science, Aarhus University, Agro Food Park 48, 8200 Aarhus N, Denmark; \\ uksundekilde@food.au.dk (U.K.S.); hannec.bertram@food.au.dk (H.C.B.) \\ 2 Department of Food Science and Technology, University of California, One Shields Avenue, Davis, CA 95616, \\ USA; mxmaldonado@ucdavis.edu \\ 3 Arla Food Ingredients, Sønderhøj 10, 8260 Viby J, Denmark; hejan@arlafoods.com \\ 4 Department of Food Science, University of Copenhagen, Rolighedsvej 26, 1958 Frederiksberg C, Denmark; \\ dn@food.ku.dk \\ * Correspondence: loujak@food.au.dk; Tel.: +45-20731316
}

Received: 19 March 2020; Accepted: 22 April 2020; Published: 24 April 2020

\begin{abstract}
Oligosaccharides from human or bovine milk selectively stimulate growth or metabolism of bacteria associated with the lower gastrointestinal tract of infants. Results from complex infant-type co-cultures point toward a possible synergistic effect of combining bovine milk oligosaccharides (BMO) and lactose (LAC) on enhancing the metabolism of Bifidobacterium longum subsp. longum and inhibition of Clostridium perfringens. We examine the interaction between $B$. longum subsp. longum and the commensal Parabacteroides distasonis, by culturing them in mono- and co-culture with different carbohydrates available. To understand the interaction between BMO and lactose on B. longum subsp. longum and test the potential postbiotic effect on C. perfringens growth and/or metabolic activity, we inoculated $C$. perfringens into fresh media and compared the metabolic changes to $C$. perfringens in cell-free supernatant from B. longum subsp. longum fermented media. In co-culture, B. longum subsp. longum benefits from $P$. distasonis (commensalism), especially in a lactose-rich environment. Furthermore, B. longum subsp. longum fermentation of BMO + LAC impaired C. perfringens' ability to utilize $\mathrm{BMO}$ as a carbon source (potential postbiotic effect).
\end{abstract}

Keywords: gut microbiota; infant nutrition; microbial interaction; co-culture; metabolic activity

\section{Introduction}

The infant immune system relies on human milk components for protection against infections [1]. Sialylated oligosaccharides from human milk protect against pathogens in the intestine of breastfed infants through synergistic mechanisms with the gut microbiota by stimulation of bifidobacteria and Bacteroides in the gut [1] and through prevention of pathogen adhesion to the intestinal surface [2]. Sialylated oligosaccharides are abundant in human colostrum and mature milk [2], but decrease with lactational age [3]. Infant formula contains only trace amounts of oligosaccharides [2], leading to microbiome differences between breast-fed and formula-fed infants. Bovine milk oligosaccharides (BMOs) are a rich source of sialylated oligosaccharides, especially $3^{\prime}$-sialyllactose (3'-SL), but also $6^{\prime}$-sialyllactose (6'-SL) [2]. The infant gut microbiome is dominated by bifidobacteria and lactobacilli, but also harbor commensals such as Bacteroides/Parabacteroides [4-7]. Several studies provide evidence of positive interactions between selected bifidobacteria and Bacteroides/Parabacteroides species [8-10] but it remains poorly understood how these interactions are linked to the specific source of fermentable 
carbohydrates. Elucidating the role of BMOs in modulating the infant microbiome composition might pave the way for using BMOs as a safe ingredient in infant formula. In a recent study [11], we found carbohydrate-dependent effects in complex co-cultures of the pathogenic Clostridium perfringens and Parabacteroides distasonis or Bifidobacterium longum subsp. longum. P. distasonis growth was positively associated with access to bovine milk oligosaccharides (BMO) and B. longum subsp. longum growth was positively associated to a combination of $\mathrm{BMO}$ and lactose (LAC). In addition, a combination of lactose and BMO negatively influenced C. perfringens growth in co-culture. Consequently, we speculate that there is a synergistic effect of combining BMO and lactose, which stimulates B. longum subsp. longum growth and simultaneously inhibits $C$. perfringens growth in a complex co-culture. In the present study, we aimed to investigate the potential interaction between B. longum subsp. longum and the commensal $P$. distasonis and the effect of carbohydrate availability by culturing them in mono- and co-culture. In addition, we aim to decipher the role of $\mathrm{BMO}$ and lactose in B. longum subsp. longum mediated inhibition of $C$. perfringens by inoculating $C$. perfringens into a cell-free supernatant of $B$. longum subsp. longum fermented lactose and BMO, i.e., elucidating any potential postbiotic effect on $C$. perfringens metabolism. Postbiotics effects include, among other microbe-mediated host-beneficial effects: the local, antimicrobial effects by e.g., short chain fatty acids and organic acids [12].

\section{Results and Discussion}

Undigested carbohydrates from human or bovine milk have a profound influence on the complex interplay between bacteria associated with the lower gastrointestinal tract of infants $[11,13,14]$. Here, we investigated a possible interaction between B. longum subsp. longum and the gut commensal $P$. distasonis when grown in co-culture. Finally, we examined the potential synergistic effect of lactose and $\mathrm{BMO}$ on $B$. longum subsp. longum growth and metabolic activity and the potential postbiotic inhibition of $C$. perfringens in cell-free media from B. longum subsp. longum. Annotated, representative ${ }^{1} \mathrm{H}$ NMR spectra of the three bacteria included in the present study are shown in Figure 1, using the $\mathrm{BMO}+\mathrm{LAC}$ treatment as an example.

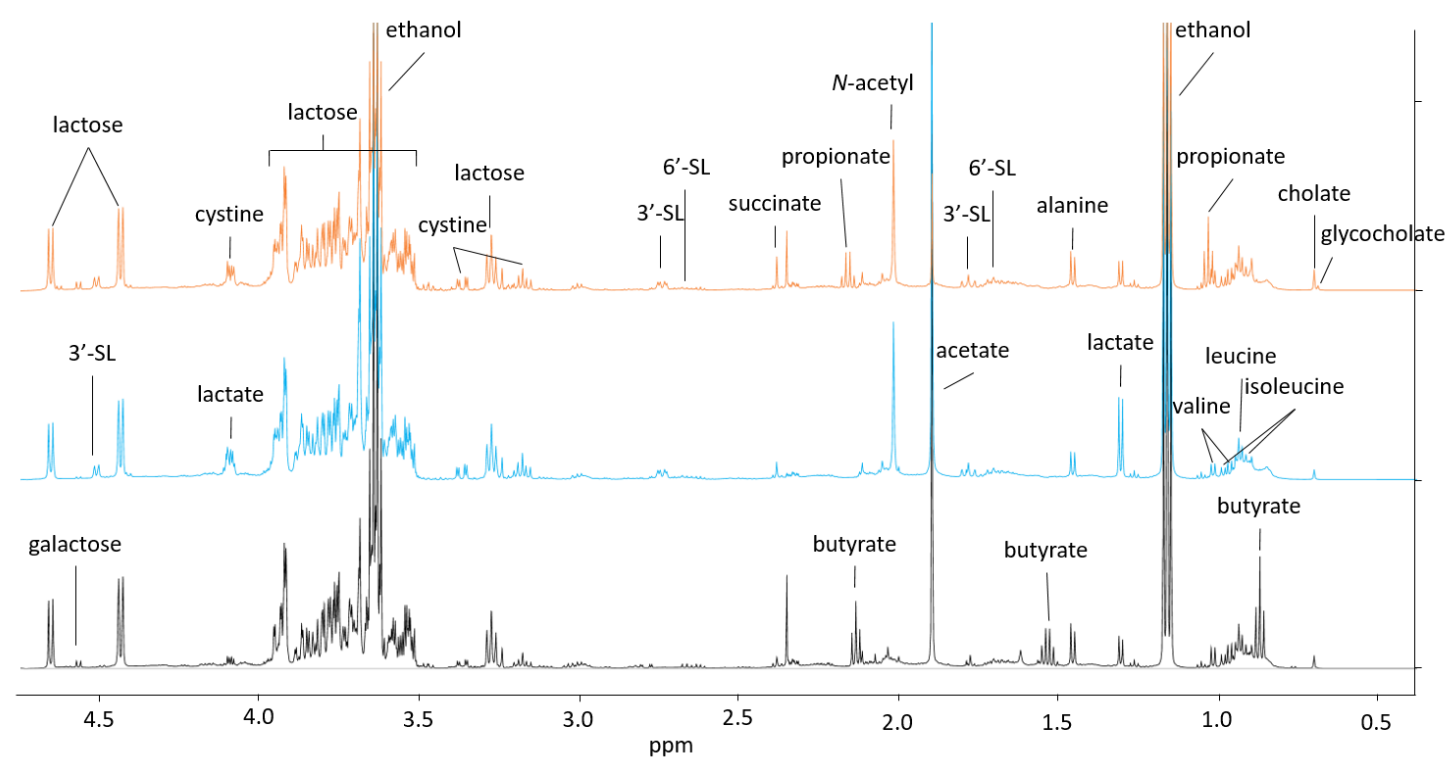

Figure 1. Aliphatic region of representative ${ }^{1} \mathrm{H}$ NMR spectra obtained for supernatant from $24 \mathrm{~h}$ fermentation of minimal media containing $1 \% \mathrm{BMO}+\mathrm{LAC}$. The colors of the spectra indicate the inoculated bacteria; orange (top): Parabacteroides distasonis, blue (middle): Bifidobacterium longum subsp. longum and black (bottom): Clostridium perfringens. BMO: bovine milk oligosaccharides, LAC: lactose. 


\subsection{A Potential Synergistic Effect of BMO and Lactose on Stimulation of B. longum subsp. longum Metabolism}

Analysis of the purified BMO product showed that $3^{\prime}$-SL and 6'-SL were the main components (37 and 6\% $w / w$ respectively), while other oligosaccharides and lactose were only present in trace amounts (data not shown). In the BMO treatment, B. longum subsp. longum does not appear to utilize $3^{\prime}$-SL or 6'-SL or produce major metabolite quantities (Supplemental Figure S1, Supplemental Table S1), but it does show growth compared to minimal media (Figure 2) after 24 and $48 \mathrm{~h}$ (except co-culture after $24 \mathrm{~h})$.

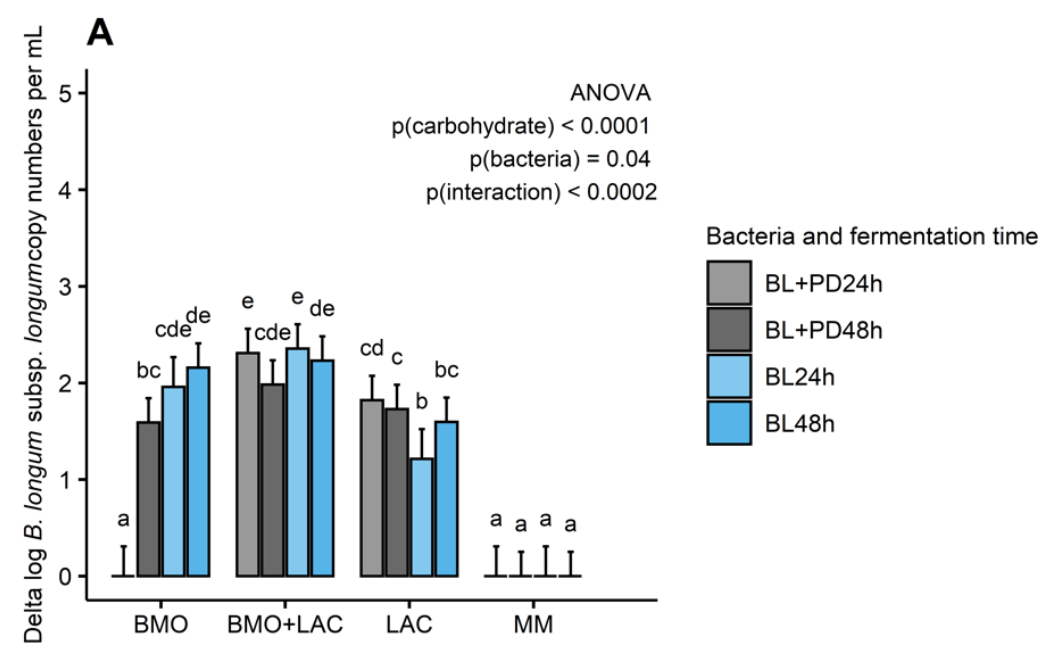

B

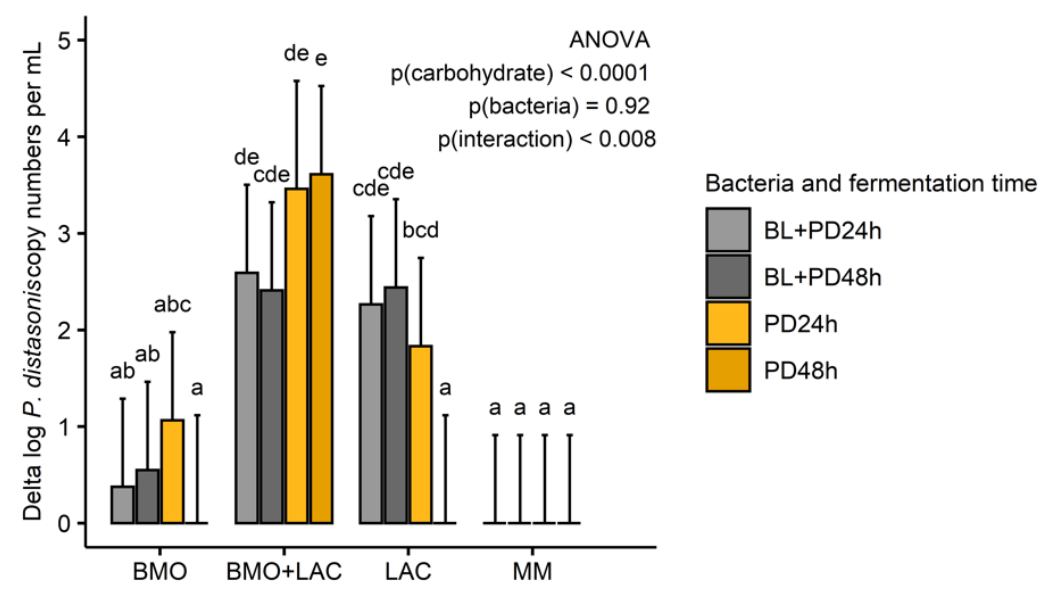

Figure 2. Delta growth of B. longum subsp. longum (A) and P. distasonis (B) in log10 copy numbers/mL culture compared to minimal media after 24 and $48 \mathrm{~h}$ fermentation of $1 \% \mathrm{BMO}, \mathrm{BMO}+\mathrm{LAC}$, or LAC media by B. longum subsp. longum (BL,-), P. distasonis (PD,-), and co-culture of B. longum subsp. longum and P. distasonis (BL $+\mathrm{PD})$. Bars represent mean values and error bars represent standard error and are based on three biological replicates and two technical replicates. Significant effect of carbon source and bacteria on delta log copy numbers/mL culture was tested by ANOVA and Tukey's HSD (honestly significant difference) was used for multiple comparisons between groups. $p \leq 0.05$ indicates significant differences and different letters in each plot indicate significant differences. BMO: bovine milk oligosaccharides, LAC: lactose, MM: minimal media (no carbohydrate source).

Unlike B. longum subsp. infantis (ATCC 15697), B. longum subsp. longum (ATCC 15707) does not have the genes necessary for expression of a sialidase [15]. Based on previous research, the inability of utilizing $3^{\prime}$-SL and $6^{\prime}$-SL is a common trait in other infant strains of B. longum subsp. longum [16]. As expected, B. longum subsp. longum is able to ferment LAC as indicated by the conversion of lactose 
carbon into acetate and formate (Supplementary Figure S1, Supplementary Table S1) and positive growth (Figure 2).

Interestingly, the B. longum subsp. longum metabolite production seems to be enhanced in the presence of both BMO and LAC. This observation is based on higher conversion of lactose into acetate, formate, and lactate (Figure 3), indicating a positive feedback mechanism on lactose utilization when in a BMO-rich environment. In addition to this, we speculate whether the higher metabolite production might be a result of $B$. longum subsp. longum metabolism of other less abundant oligosaccharides in the BMO product. B. longum subsp. longum isolates grew well on lacto-N-tetraose and lacto-N-neotetraose [16], neutral HMOs, that are found in minor amounts in bovine milk [17], but not quantified in the current study. According to the complete genome, B. longum subsp. longum ATCC 15707 (GenBank accession number AP010888.1) contains annotated genes for hydrolysis of neutral oligosaccharides with type I core structure (endo- $\alpha-\mathrm{N}$-acetylgalactosaminidase) and putative $\beta$-hexosaminidases. More work is needed to elucidate the impact of $\mathrm{BMO}$ components on lactose and oligosaccharide utilization in B. longum subsp. longum (ATCC 15707).

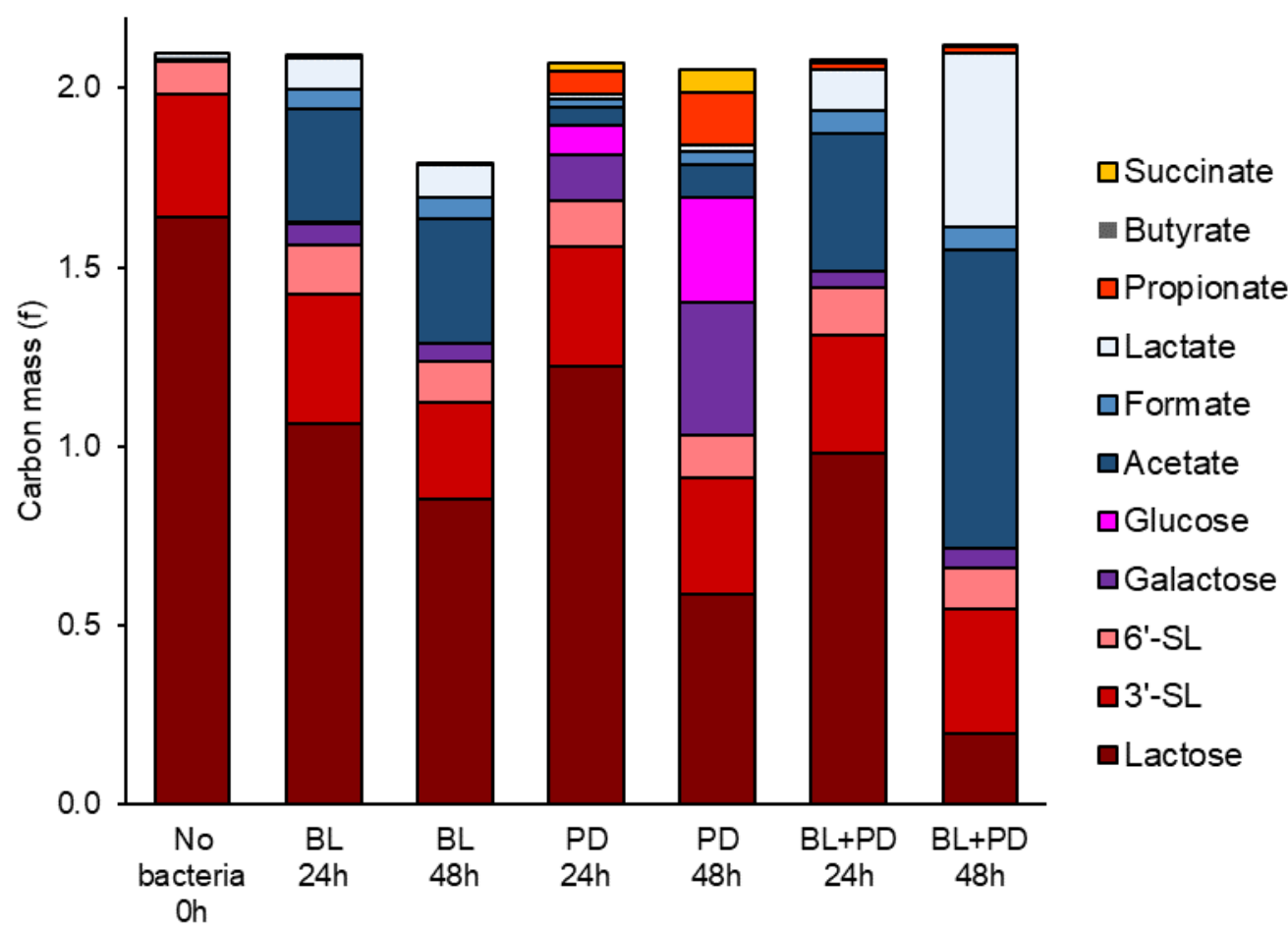

Figure 3. Carbon mass balance after 24 and $48 \mathrm{~h}$ fermentation of $1 \% \mathrm{BMO}+\mathrm{LAC}$ media by B. longum subsp. longum (BL), P. distasonis (PD) or co-culture of B. longum subsp. longum (BL, PD). Carbon mass was calculated as $g$ substrate $\times$ number of $C$-atoms $\times$ molecular mass of $C(12.01 \mathrm{u})$ divided by the molecular weight of substrate. Each stacked segment of the bars represents a mean value from three biological replicates. BMO: bovine milk oligosaccharides, LAC: lactose.

In a previous study, pure $\mathrm{BMO}$ appeared to stimulate $P$. distasonis growth in a complex infant-type co-culture [11]. Little is known about sialic acid utilization by $P$. distasonis, but a comparison of genomic sequences to Bacteroides fragilis revealed sequence similarities in the nanLET gene cluster and encoding of a nanH homolog (sialidase) [18]. The genome of P. distasonis ATCC 8503 (GenBank accession number AB238922) contains one candidate sialidase and one putative exo- $\alpha$-sialidase as well as several candidate $\beta-\mathrm{N}$-acetylhexosaminidases. Consequently, $P$. distasonis should be able to hydrolyze sialic acid from siallylactose and hexoses from neutral oligosaccharides and utilize them as carbon and energy sources when grown on BMO and BMO + LAC. P. distasonis cultures with BMO showed an average increase of $1.06 \mathrm{logs}$ after 24 , while $48 \mathrm{~h}$ growth was not above detection limit. However, 
due to the large difference in the maximum cell numbers reached in each replicate, overall growth did not reach significance (Figure 2). Nonetheless, $P$. distasonis releases minor amounts of lactose to the supernatant (Supplementary Figure S2 and Supplementary Table S2), which is an indication of utilization of lactose-bound carbon. The P. distasonis (ATCC 8503) genome contains candidate $\beta$-galactosidases, and indeed, in LAC fermentations, $P$. distasonis hydrolyzes lactose, which is evident in the glycan data, where it releases galactose and glucose to the supernatant (Supplementary Figure S1, Supplementary Table S1).

\subsection{P. distasonis and B. longum subsp. longum Co-Culture Increase Utilization of Lactose}

Previous results from co-cultures of multiple organisms including P. distasonis and B. longum subsp. longum have suggested a substrate-dependent relationship between these two bacteria [11]. The current data suggests that commensalism occurs between B. longum subsp. longum and P. distasonis, as indicated by the changes in concentration of glycans in the supernatant of mono- and co-culture fermentation of LAC and BMO + LAC. Figure 3 presents the glycan and short-chain fatty acid composition as carbon mass. It is evident from carbon mass of glycan and short-chain fatty acids that growth of B. longum subsp. longum + P. distasonis co-culture during fermentation of LAC (Supplementary Figure S1, Supplementary Table S1) and BMO + LAC media (Figure 3, Supplementary Table S3), result in a higher proportion of lactose carbon converted into acetate, formate, and lactate, indicating higher metabolic activity of B. longum subps. longum in co-culture (BL + PD) compared to mono-culture (BL or PD). This is especially evident in BMO + LAC treatment (Figure 3). While P. distasonis appears to hydrolyze lactose extracellularly as indicated by the release of galactose and glucose into the supernatant (Supplementary Figure S1, Supplementary Table S1), B. longum subsp. longum most likely transports lactose into the cell and metabolizes it intracellularly. Interestingly, it appears that B. longum subsp. longum + P. distasonis co-cultures enhance fermentation of LAC and positively influences B. longum subsp. longum growth in $24 \mathrm{~h}$ co-culture samples (Figure 2). Even though B. longum subsp. longum is capable of utilizing galactose and glucose monomers released by $P$. distasonis, it is likely that they are not the preferred substrate when lactose is also available in the media. This is in accordance with data from another B. longum subsp. longum strain (NCC2705) that showed a preference for lactose rather than glucose as a substrate for carbon and energy metabolism, which could directly be traced back to lactose repression of the glucose-specific permease (glcP) [19]. The higher concentration of acetate and lactate in the co-culture with enhanced fermentation of LAC (Supplementary Figure S1, Supplementary Table S1) is also an indication of enhanced B. longum subsp. longum metabolism. Fermentation of BMO + LAC by B. longum subsp. longum shows a similar metabolic pattern as in the fermentation of LAC, but the concentrations of acetate and lactate become even higher (Figure 3, Supplemental Figures S1 and S2, Supplemental Tables S1-S3): this is especially pronounced in the co-cultures. Growth of B. longum subsp. longum or P. distasonis are not significantly different between mono- or co-culture fermentation of BMO + LAC (Figure 2). B. longum subsp. longum and P. distasonis mono- and co-cultures show very low metabolic activity when grown solely on BMO as indicated by low SCFA production and (very) limited change in glycans (Supplementary Table S4).

\subsection{Through Metabolism of BMO + LAC B. longum subsp. longum Potentially Inhibits C. perfringens' Ability to Metabolize Sialyllactose}

C. perfringens was able to utilize BMO and LAC for growth. C. perfringens completely depleted $3^{\prime}$-SL and $6^{\prime}$-SL in the BMO media (Figure 4A,B), with a concomitant minor increase in lactose content (Figure $4 \mathrm{C}$ ).

This finding indicates that $C$. perfringens utilizes the sialic acid monomer by a sialidase acting on the 2,3 and 2,6 glycosidic bonds of the sialyllactose units with subsequent lactose release. It is known that most bacterial pathogens carry genes encoding for sialidases to be able to harvest sialic acid from mucus [20]. Bacterial utilization of sialic acid from sialyllactose requires a sialidase, which is encoded by NanH, NanI, NanJ, or orthologous genes [21]. Previous studies found what appears to be a highly 
conserved sialidase encoding ORF (open reading frame) in many strains of C. perfringens [22,23] and, in fact, according to the sequence and annotated genome of C. perfringens ATCC $13124^{\mathrm{T}}$ (GenBank accession number CP000246) does indeed encode three sialidases (nanH, nanI, and nanJ). C. perfringens fermentation of LAC resulted in a lower lactose concentration compared to uninoculated media (-,-) (Figure 4C) and minor increase in galactose (Figure 4D). The genome of C. perfringens ATCC 13124 (GenBank accession number CP000246) contains annotated $\beta$-galactosidase, sugar transporters, and sugar ABC transporters. Collectively our data and literature indicate a selective utilization of the glucose monomer from lactose, while uptake of lactose or galactose needs further investigations to decipher the catabolism pathways.

A



D

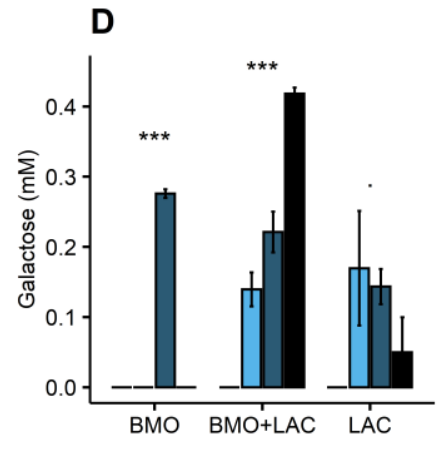

B

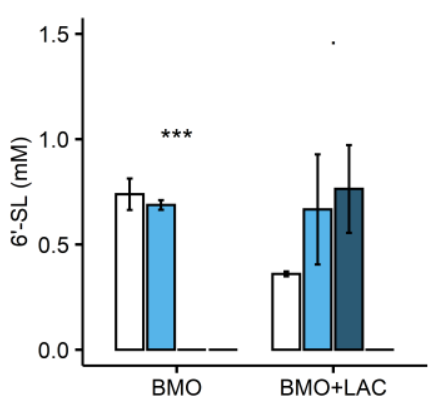

E

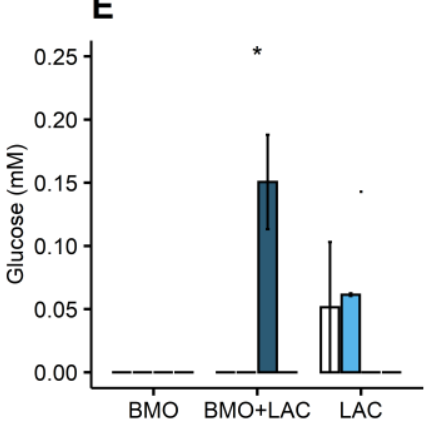

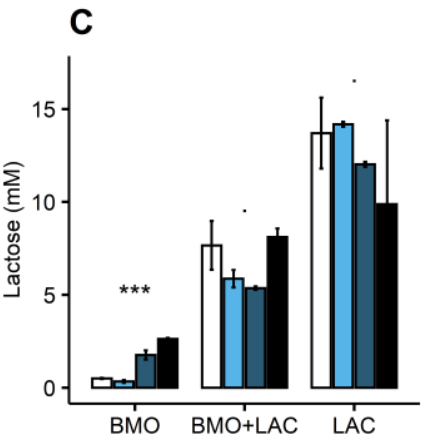

Bacteria

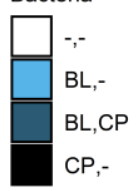

Figure 4. Glycan concentration ( $\mathrm{mM})$ in $1 \% \mathrm{BMO}, \mathrm{BMO}+\mathrm{LAC}$, and LAC media in uninoculated media (-,-) and after $24 \mathrm{~h}$ fermentation by B. longum subsp. longum (BL,-) or C. perfringens (CP,-) and C. perfringens fermentation of cell-free media from B. longum subsp. longum (BL,CP). (A) $3^{\prime}$-sialyllactose, (B) 6'-sialyllactose, (C) lactose, (D) galactose, (E) glucose. Bars represent mean values and error bars represent standard error and are based on two biological replicates. Stars indicate statistical significance by $p$-values from ANOVA testing on the bacteria effect within each carbohydrate treatment: $p<0.001:{ }^{* * *}, p<0.01:{ }^{*}, p<0.1$. BMO: bovine milk oligosaccharides, LAC: lactose.

Growth (Figure 5) and butyrate production (Figure 6B) support the finding that $C$. perfringens is capable of utilizing BMO and lactose components as carbon sources. Depletion of $3^{\prime}$-SL and $6^{\prime}$-SL (Figure 4A,B) and a minor increase in galactose (Figure 4D) suggest that $C$. perfringens utilizes all the sialic acid and some lactose-derived glucose in the BMO + LAC treatment. Despite notable C. perfringens growth and metabolism in BMO and LAC treatments, the combination of $\mathrm{BMO}+\mathrm{LAC}$ resulted in $C$. perfringens growth that was not significantly different from growth on minimal medium (Figure 5). However, the lower concentration of butyrate and acetate are indicative of a lower metabolic activity of $C$. perfringens in the BMO + LAC treatment (Figure 6A,B) compared to the pure BMO or LAC treatments. This cannot be attributed to acid accumulation generated during C. perfringens metabolism, as $\mathrm{pH}$ was comparable in $C$. perfringens fermentation of BMO + LAC (6.19 \pm 0.23$), \mathrm{BMO}(6.06 \pm 0.03)$, and LAC (6.37 \pm 0.69$)$. Most likely, the lower growth of $C$. perfringens is a result of a lower total carbon concentration in the BMO + LAC media, as the BMO component contained only $44 \%$ oligosaccharides. 


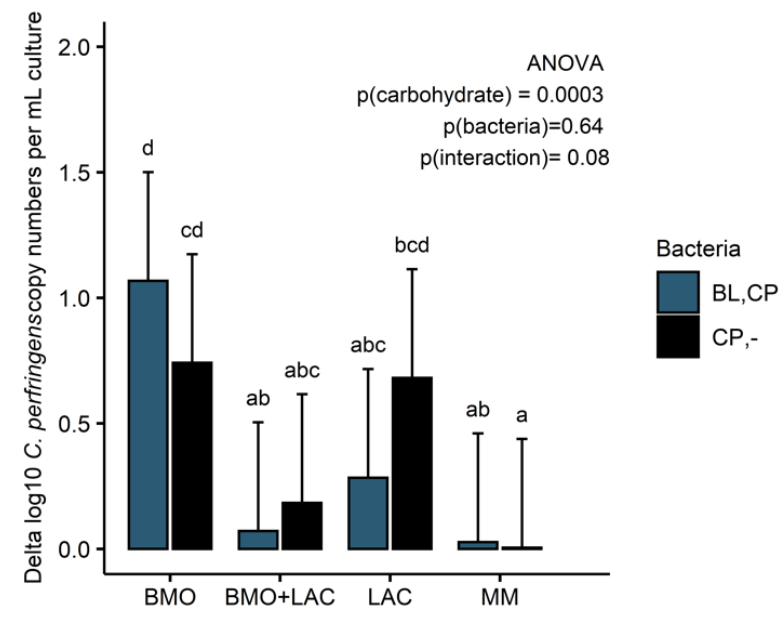

Figure 5. Delta $C$. perfringens growth in log10 copy numbers/mL culture compared to minimal media after $24 \mathrm{~h}$ fermentation of fresh 1\% BMO, BMO + LAC, and LAC media (CP,-) and fermentation by C. perfringens in cell-free media obtained after $24 \mathrm{~h}$ fermentation by B. longum subsp. longum (BL,CP). Bars represent mean values and error bars standard error and are based on two biological replicates and two technical replicates. Significant effect of carbon source and bacteria on delta log copy numbers $/ \mathrm{mL}$ culture was tested by ANOVA and Tukey's HSD (honestly significant difference) was used for multiple comparisons between groups. $p \leq 0.05$ indicates significant differences and different letters in each plot indicate significant differences. BL: B. longum subsp. longum, CP: C. perfringens, BMO: bovine milk oligosaccharides, LAC: lactose. MM: minimal media (no carbohydrate).
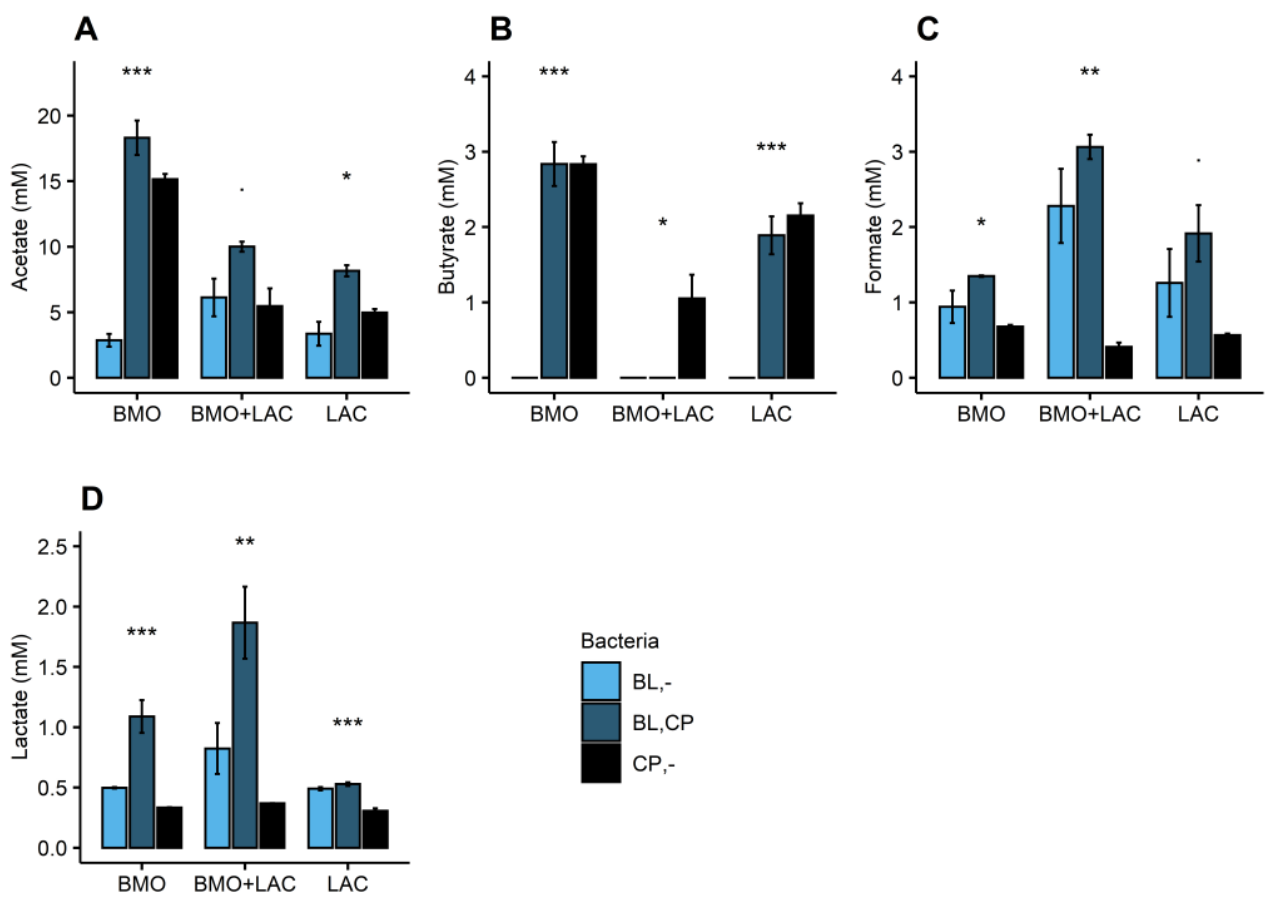

Figure 6. Metabolite concentration ( $\mathrm{mM})$ of $(\mathbf{A})$ acetate, $(\mathbf{B})$ butyrate, $(\mathbf{C})$ formate, and $(\mathbf{D})$ lactate after $24 \mathrm{~h}$ fermentation of $1 \% \mathrm{BMO}, \mathrm{LAC}$, and BMO + LAC media by B. longum subsp. longum (BL,-), C. perfringens $(\mathrm{CP},-)$ or $C$. perfringens fermentation in cell-free media obtained after $24 \mathrm{~h}$ fermentation by $B$. longum subsp. longum (BL,CP). Bars represent mean values and error bars represent standard error and are based on two biological replicates. Stars indicate statistical significance by $p$-values from ANOVA testing on the bacteria effect within each carbohydrate treatment: $p<0.001$ : ***, $p<0.01:{ }^{*}, p<0.05:{ }^{*}, p<0.1$. BL: B. longum subsp. longum, CP: C. perfringens, BMO: bovine milk oligosaccharides, LAC: lactose. 
The potential synergy between BMO and lactose on B. longum subsp. longum metabolism is of considerate interest. Not only does BMO + LAC increase metabolic activity of B. longum subsp. longum, but additionally the cell-free media from $B$. longum subsp. longum fermentation of $B M O+$ LAC appeared to inhibit $C$. perfringens' ability to utilize sialic acid from $6^{\prime}$-SL or 3'-SL (Figure 4A,B). The absence of butyrate in the resulting $C$. perfringens supernatant (Figure $6 \mathrm{~B}$ ) indicates a decrease in the metabolic activity of $C$. perfringens. This suggests a B. longum subsp. longum-metabolite or $\mathrm{pH}$ mediated inhibition of $C$. perfringens' capacity to obtain sialic acid from 3'-SL and 6'SL, possibly through direct or indirect effects on the sialidase activity or expression. The $\mathrm{pH}$ of the cell-free media B. longum subsp. longum fermentation of BMO + LAC was low $(5.42 \pm 0.30)$ and even lower after C. perfringens fermentation $(4.98 \pm 0.14)$. The activity of $C$. perfringens sialidases are $\mathrm{pH}$-sensitive, but the optimum is around $\mathrm{pH} 5-5.5$ [24,25], and we therefore question if $\mathrm{pH}$ is the direct reason for inhibited sialidase activity. Sialidase production in C. perfringens is complicated, but it appears to be indirectly regulated by the VirS/VirR system, which is also an important regulator or virulence genes in C. perfringens. As an alternate hypothesis, we speculate that the low $\mathrm{pH}$ and organic acids in B. longum subsp. longum cell-free media, decreases sialidase expression through effects on the virulence gene expression. In Clostridium difficile, the virulence gene expression is $\mathrm{pH}$-sensitive (lower expression at lower $\mathrm{pH})[22,23,26]$. If there is a similar $\mathrm{pH}$-dependent effect on virulence gene expression in C. perfringens, a low $\mathrm{pH}$ might have downstream effects on sialidase production.

\section{Materials and Methods}

\subsection{Strains and Propagation}

This study included three bacterial strains: Parabacteroides distasonis (ATCC 8503), Clostridium perfringens (ATCC 13124), and Bifidobacterium longum subsp. longum (ATCC 15707). Frozen stocks were activated and propagated in rich media: B. longum supsp. longum in MRS (Thermo Scientific Oxoid, Waltham, MA, USA) with 0.05\% L-cysteine and C. perfringens and P. distasonis in Brain Heart Infusion (Thermo Scientific Oxoid, Waltham, MA) in an anaerobic chamber operating at $37^{\circ} \mathrm{C}$ with the following gas composition: $7 \% \mathrm{CO}_{2} / 3 \% \mathrm{H}_{2} / 90 \% \mathrm{~N}_{2}$. Growth curves under anaerobic conditions of each strain was determined at $37^{\circ} \mathrm{C}$ by measuring optical density at $600 \mathrm{~nm}$ in an automated plate reader (BioTek, Winooski, VT, USA) for microtiter plates placed in an anaerobic chamber.

\subsection{Lactose and BMO Treatments}

This study included two carbohydrate sources: pure lactose and purified bovine milk oligosaccharides (BMO, Arla Food Ingredients Group P/S, Aarhus, Denmark). The BMO and minimal media composition was described in detail elsewhere [11]. Minimal media with $1 \%(w / v)$ lactose (termed LAC), BMO (termed BMO), or BMO and lactose (termed BMO + LAC) as the sole carbohydrate source were used for fermentation experiments. The media were pre-reduced by placing in an anaerobic chamber at room temperature overnight.

\subsection{Standard Curves}

Standard curves were obtained by inoculating a single colony into fresh rich media and incubated at $37^{\circ} \mathrm{C}$ in a heating cabinet placed in an anaerobic chamber. Cells were harvest at the late exponential phase to maximize the $\mathrm{CFU} / \mathrm{mL}$ and minimize dead cells. Standard curve cultures were diluted $10^{-4}-10^{-7}$ in sterile saline $(0.9 \% \mathrm{NaCl})$, plated onto agar plates and incubated anaerobically at $37^{\circ} \mathrm{C}$ until counts were stable (48-72 h) and $\log \mathrm{CFU} / \mathrm{mL}$ calculated using the average count of colonies for the most populated plates with counts $>20$ and $<250$. The undiluted pure cultures were frozen at $-80{ }^{\circ} \mathrm{C}$ for later DNA extraction and qPCR quantification. 


\subsection{Fermentation of Lactose and BMO in Mono- and Co-Culture}

This study consists of three sub-experiments: (1) mono-cultures of P. distasonis, B. longum subsp. longum, and C. perfringens in LAC, BMO, or BMO + LAC (24 h), (2) co-culture of P. distasonis and B. longum sups. longum in 1\% carbohydrate media (24 and $48 \mathrm{~h}$ ), (3) mono-culture of C. perfringens in cell-free media from $P$. distasonis and B. longum subsp. longum after $24 \mathrm{~h}$ fermentation of LAC, BMO, and $\mathrm{BMO}+\mathrm{LAC}$. Late-log cultures were washed and adjusted to optical density $1.0\left(\mathrm{OD}_{600 \mathrm{~nm}}\right)$ using sterile saline $(0.9 \%(w / v) \mathrm{NaCl})$ before inoculation. The co-culture inoculum consisted of OD-adjusted inoculum of P. distasonis and B. longum subsp. longum. Mono- and co-cultures contained 1\% inoculum in pre-reduced media. Sterile water served as the negative control. Mono-culture experiments included two biological replicates, while co-cultures included three. After fermentation, samples were centrifuged at $7500 \mathrm{~g}$ for $1 \mathrm{~min}$ to produce supernatant and pellet samples. Sterile-filtered (using $0.22 \mu \mathrm{m}$ syringe filters), cell-free media from mono-cultures of P. distasonis and B. longum sups. longum was re-inoculated with $C$. perfringens inoculum and allowed to propagate for $24 \mathrm{~h}$. Sterile-filtered supernatant samples and pellets were frozen at $-80^{\circ} \mathrm{C}$ until further analysis.

\subsection{Proton Nuclear Magnetic Resonance $\left({ }^{1} \mathrm{H}\right.$ NMR) Metabolomics}

Supernatant samples were thawed for $30 \mathrm{~min}$, vortexed, and filtered through prewashed 10k Millipore centrifugal filters (Amicon Ultra, Millipore Corp., Billerica, MA, USA) by centrifugation at $14,000 \times \mathrm{g}$ for $30 \mathrm{~min}$ at $4{ }^{\circ} \mathrm{C}$. A volume of $500 \mu \mathrm{L}$ of filtered sample was transferred to an NMR tube with $60 \mu \mathrm{L}$ of phosphate buffer $(\mathrm{pH}=7.4)$ containing $0.23 \mathrm{mM}$ DSS (3-(trimethylsilyl)-1-propanesulfonic acid-d6 sodium salt, Sigma-Aldrich, St. Louis, MO, USA) and $70 \mu \mathrm{L}$ of $\mathrm{D}_{2} \mathrm{O}$ (deuterium oxide, 99.9\%, Cambridge Isotope Laboratories, Andover, MA, USA). NMR spectra were acquired on a Bruker Avance $600 \mathrm{MHz}$ NMR spectrometer (Bruker BioSpin, Gmbh, Rheinstetten, Germany) operating at a proton NMR frequency of $600.13 \mathrm{MHz}$ and equipped with a $5 \mathrm{~mm}$ TXI probe. The 1D NOESY pulse experiment with pre-saturation of the spectral region containing the water peak (noesypr1d) was used with a recycle delay of $5 \mathrm{~s}$. A total of 64 FIDs were acquired, and the acquisition parameters included $32 \mathrm{~K}$ complex data points, a spectral width of $7289 \mathrm{~Hz}(12.15 \mathrm{ppm})$, and an acquisition time of $2.25 \mathrm{~s}$. Measurements were done at $298 \mathrm{~K}\left(25^{\circ} \mathrm{C}\right)$.

\subsection{Preprocessing of ${ }^{1} \mathrm{H}$ NMR Data for Multivariate Analysis}

An experimental window function with a line-broadening factor of $0.3 \mathrm{~Hz}$ was applied to all FIDs before Fourier transformation. The resulting spectra were manually phase corrected and automatically baseline corrected by polynomials using the Topspin 3.0 software (Bruker BioSpin, Gmbh, Rheinstetten, Germany). In Matlab (Version R2016a, The MathWorks Inc., Natic, MA, USA), data were referenced and scaled according to DSS at $0.0 \mathrm{ppm}$. Metabolites of interest were quantified using the Chenomx NMR suite software (Version 8.1 professional, Edmonton, AB, Canada) using the build-in library and an in-house library of $3^{\prime}$-SL and 6' -SL standards (Carbosynth, Berkshire, UK).

\subsection{Carbon Mass in Co-Culture Experiment}

Carbon mass was calculated for selected glycans and short-chain fatty acids using the following equation: $g$ substrate $\times$ number of $\mathrm{C}$-atoms $\times$ molecular mass of carbon/molecular weight of the substrate, as presented in [27]. The calculations were performed in Excel (Microsoft Office Professional Plus 2016).

\subsection{DNA Extraction}

Genomic DNA was extracted from bacterial pellets using the DNeasy Blood and Tissue kit (Qiagen, Valencia, CA, USA) according to manufacture protocol. Briefly, bacterial pellets were re-suspended in lysozyme solution and incubated at $37^{\circ} \mathrm{C}$ for $30 \mathrm{~min}$ followed by Proteinase $\mathrm{K}$ treatment in AL buffer at $56^{\circ} \mathrm{C}$ for $30 \mathrm{~min}$. After addition of ethanol and vortex mixing the remaining steps were conducted 
according to the manufacturer's instructions on DNA extraction from animal tissues. The extracted DNA was diluted 1:10 in RNAse free water and stored at $-20^{\circ} \mathrm{C}$ until further use.

\subsection{Quantitative PCR}

Quantitative PCR was performed using primers specific for the 16S rRNA gene of each of the three bacterial strains. The primers were previously described in [11]. Primers were ordered from Integrated DNA Technologies Inc. (San Diego, CA, USA). The bacterial DNA from the pure cultures were used as positive control and serial diluted in sterile Milli-Q water $\left(10^{-1}-10^{-6}\right)$ to produce standard curves. The qPCR assay was run with a master mix containing forward $(10 \mathrm{pmol})$ and reverse $(20 \mathrm{pmol})$ primers, RNAse free water, and PowerUp SYBR Green Master Mix (Life Technologies, Carlsbad, CA, USA). A total of $3 \mu \mathrm{L}$ of DNA or RNase free water for non-template controls (NTC) were added to each well to reach a final reaction volume of $20 \mu \mathrm{L}$. Two technical replicates were included per biological sample. The qPCR assay was run on a 7500 Fast Real-Time PCR System (Life Technologies, Dublin, Ireland), using the following program: $50{ }^{\circ} \mathrm{C}(2 \mathrm{~min})$, at $95^{\circ} \mathrm{C}(2 \mathrm{~min}), 40$ cycles of: $95^{\circ} \mathrm{C}(15 \mathrm{~s}), 56^{\circ} \mathrm{C}$ $(15 \mathrm{~s}), 72{ }^{\circ} \mathrm{C}(1 \mathrm{~min})$. A melting curve analysis at $95^{\circ} \mathrm{C}(15 \mathrm{~s}), 56{ }^{\circ} \mathrm{C}(1 \mathrm{~min}), 95^{\circ} \mathrm{C}(30 \mathrm{~s})$, and $56{ }^{\circ} \mathrm{C}$ $(15 \mathrm{~s})$ was performed to estimate the melting temperature of the amplicons. Melting curves with more than the expected single peak indicate unspecific PCR products and primer-dimer formation. The detection limit for inclusion of samples in the data analysis was determined to be $10^{6}$ copy numbers $/ \mathrm{mL}$ culture. Delta log copy numbers per $\mathrm{mL}$ culture were calculated by subtracting the mean value of log copy numbers per $\mathrm{mL}$ in minimal media from each replicate value of log copy numbers per $\mathrm{mL}$ in carbohydrate media for each type of bacteria.

\subsection{Data Analysis}

Metabolite and glycan concentrations (mM) were presented as mean values with error bars representing standard error in bar charts. One-way ANOVA was applied to test the effect of bacteria on metabolite and glycan concentrations within each carbohydrate treatment.

In the co-culture study, growth data (delta log copy numbers per mL culture) were analyzed using two-way ANOVA to test the effect of carbohydrate treatment, bacteria (mono- or co-culture) as well as the interaction on growth. In the C. perfringens experiments, a two-way ANOVA was used to test the effect of carbohydrate and pretreatment of the media with B. longum subsp. longum as well as the interaction effect. All growth data are presented in barplots with lsmeans as bars and error bars representing standard error, as produced by an lsmeans fitted model. Tukey's test for multiple comparisons was used to test the difference between treatment groups using a significance level of $\alpha=0.05$.

Before ANOVA analysis of metabolite or growth data, the datasets were checked for variance homogeneity and normality by visual inspection of Residual vs. Fitted and QQplots, respectively. Deviating samples were removed only if it could be argued they were indeed deviating substantially from e.g., other replicates of the same sample and if the sample contributed to non-normality of the dataset. Data analysis and visualization was performed in R (version 3.6.1, The R Foundation, Vienna, Austria).

Data is deposited as supplementary material.

\section{Conclusions}

In conclusion, the present study points toward a potential synergistic effect of lactose and BMO on metabolism of B. longum subsp. longum. Furthermore, it appears that commensalism between $B$. longum subsp. longum and $P$. distasonis in lactose-rich environment is beneficial for B. longum subsp. longum activity. Finally, metabolites from B. longum subsp. longum fermentation of BMO + LAC appeared to diminish $C$. perfringens' ability to utilize BMO as a carbon source (potential postbiotic effects). Conducting additional research using other bifidobacteria strains capable of utilizing sialyated 
oligosaccharides for energy and metabolism is of major interest due to the possibility of enhancing the metabolic effect observed in the current study.

Supplementary Materials: The following are available online at http://www.mdpi.com/2218-1989/10/4/167/s1, Experimental and research data, Figure S1: Carbon mass balance after 24 or $48 \mathrm{~h}$ fermentation of $1 \%$ LAC by Bifidobacterium longum subsp. longum (BL), Parabacteroides distasonis (PD), or co-culture (BL + PD). Carbon mass was calculated as $\mathrm{g}$ substrate $\times$ number of $\mathrm{C}$-atoms $\times 12$ divided by the molecular weight of substrate. Each stacked segment of the bars represents a mean value from three biological replicates. LAC: lactose, Figure S2: Carbon mass balance after 24 or $48 \mathrm{~h}$ fermentation of $1 \%$ BMO by Bifidobacterium longum subsp. longum (BL), Parabacteroides distasonis (PD), or co-culture (BL + PD). Carbon mass was calculated as g substrate $\times$ number of $\mathrm{C}$-atoms $\times 12$ divided by the molecular weight of substrate. Each stacked segment of the bars represents a mean value from three biological replicates. BMO: bovine milk oligosaccharides, Table S1: Carbon mass after 24 or $48 \mathrm{~h}$ fermentation of 1\% LAC by Bifidobacterium longum subsp. longum (BL), Parabacteroides distasonis (PD), or co-culture $(\mathrm{BL}+\mathrm{PD})$. Carbon mass was calculated as g substrate $\times$ number of $\mathrm{C}$-atoms $\times 12$ divided by the molecular weight of substrate. The data represent mean and SD is the standard deviation obtained from three biological replicates. LAC: lactose, Table S2: Carbon mass after 24 or $48 \mathrm{~h}$ fermentation of $1 \%$ BMO by Bifidobacterium longum subsp. longum (BL), Parabacteroides distasonis (PD), or co-culture (BL + PD). Carbon mass was calculated as g substrate $\times$ number of $\mathrm{C}$-atoms $\times 12$ divided by the molecular weight of substrate. The data represent mean and SD is the standard deviation obtained from three biological replicates. BMO: Bovine milk oligosaccharides, Table S3: Carbon mass after 24 or $48 \mathrm{~h}$ fermentation of $1 \% \mathrm{BMO}+\mathrm{LAC}$ by Bifidobacterium longum subsp. longum (BL), Parabacteroides distasonis (PD), or co-culture (BL + PD). Carbon mass was calculated as $g$ substrate $\times$ number of $\mathrm{C}$-atoms $\times 12$ divided by the molecular weight of substrate. The data represent mean and SD is the standard deviation obtained from three biological replicates. BMO: Bovine milk oligosaccharides, LAC: lactose, Table S4: Growth (log copy numbers per mL culture) of Parabacteroides distasonis (PD), Bifidobacterium longum subsp. longum (BL), and Clostridium perfringens (CP) in $1 \%$ treatments of bovine milk oligosaccharides (BMO), lactose (LAC) or a combination of the two (BMO + LAC). C. perfringens was grown on spent media from B. longum subsp. longum (BL,CP) and P. distasonis (PD,CP). Significant effect of carbon source on log CFU/mL culture was tested by ANOVA and Tukey's HSD was used for multiple comparisons between groups. $p \leq 0.05$ indicates significant differences and different letters in each row indicate significant differences.

Author Contributions: Conceptualization, L.M.A.J., M.X.M.-G., U.K.S., H.J.A., H.C.B. and D.S.N.; Data curation, L.M.A.J. and M.X.M.-G.; Formal analysis, L.M.A.J.; Funding acquisition, H.C.B.; Investigation, L.M.A.J., M.X.M.-G., U.K.S., H.J.A., H.C.B. and D.S.N.; Methodology, L.M.A.J. and M.X.M.-G.; Project administration, H.C.B.; Resources, L.M.A.J., M.X.M.-G., H.J.A. and H.C.B.; Software, L.M.A.J.; Supervision, U.K.S., H.J.A., H.C.B. and D.S.N.; Validation, L.M.A.J., M.X.M.-G., U.K.S., H.J.A., H.C.B. and D.S.N.; Visualization, L.M.A.J.; Writing-original draft, L.M.A.J.; Writing-review and editing, L.M.A.J., M.X.M.-G., U.K.S., H.J.A., H.C.B. and D.S.N. All authors have read and agreed to the published version of the manuscript.

Funding: L.M.A.J. was funded by Arla Foods Amba and Aarhus University as part of L.M.A.J.'s Ph.D project.

Acknowledgments: The authors wish to acknowledge David A. Mills at University of California, Davis for making his laboratory available and providing the materials for the microbiology part of this study.

Conflicts of Interest: The authors declare no conflict of interest. Arla Food Ingredients Group P/S provided the bovine milk oligosaccharides used in this study.

\section{References}

1. Newburg, D.S.; Walker, W.A. Protection of the neonate by the innate immune system of developing gut and of human milk. Pediatr. Res. 2007, 61, 2-8. [CrossRef] [PubMed]

2. Ten Bruggencate, S.J.M.; Bovee-Oudenhoven, I.M.J.; Feitsma, A.L.; van Hoffen, E.; Schoterman, M.H.C. Functional role and mechanisms of sialyllactose and other sialylated milk oligosaccharides. Nutr. Rev. 2014, 72,377-389. [CrossRef] [PubMed]

3. Sundekilde, U.K.; Downey, E.; O'Mahony, J.A.; O'Shea, C.-A.; Ryan, C.A.; Kelly, A.L.; Bertram, H.C. The effect of gestational and lactational age on the human milk metabolome. Nutrients 2016, 8, 304. [CrossRef] [PubMed]

4. Martin, F.-P.J.; Sprenger, N.; Yap, I.K.; Wang, Y.; Bibiloni, R.; Rochat, F.; Rezzi, S.; Cherbut, C.; Kochhar, S.; Lindon, J.C.; et al. Panorganismal gut microbiome- host metabolic crosstalk. J. Proteome Res. 2009, 8, 2090-2105. [CrossRef]

5. Martin, F.-P.J.; Wang, Y.; Sprenger, N.; Yap, I.K.S.; Lundstedt, T.; Lek, P.; Rezzi, S.; Ramadan, Z.; van Bladeren, P.; Fay, L.B.; et al. Probiotic modulation of symbiotic gut microbial-host metabolic interactions in a humanized microbiome mouse model. Mol. Syst. Biol. 2008, 4, 157-171. [CrossRef] 
6. Yassour, M.; Vatanen, T.; Siljander, H.; Hämäläinen, A.-M.; Härkönen, T.; Ryhänen, S.J.; Franzosa, E.A.; Vlamakis, H.; Huttenhower, C.; Gevers, D.; et al. Natural history of the infant gut microbiome and impact of antibiotic treatment on bacterial strain diversity and stability. Sci. Transl. Med. 2016, 8, 343ra81. [CrossRef]

7. Laursen, M.F.; Andersen, L.B.; Michaelsen, K.F.; Mølgaard, C.; Trolle, E.; Bahl, M.I.; Licht, T.R. Infant gut microbiota development is driven by transition to family foods independent of maternal obesity. Msphere 2016, 1, e00069-15. [CrossRef]

8. Rios-Covian, D.; Arboleya, S.; Hernandez-Barranco, A.M.; Alvarez-Buylla, J.R.; Ruas-Madiedo, P.; Gueimonde, M.; Clara, G. Interactions between Bifidobacterium and Bacteroides species in cofermentations are affected by carbon sources, including exopolysaccharides produced by bifidobacteria. Appl. Environ. Microbiol. 2013, 79, 7518-7524. [CrossRef]

9. Falony, G.; Calmeyn, T.; Leroy, F.; De Vuyst, L. Coculture fermentations of Bifidobacterium species and Bacteroides thetaiotaomicron reveal a mechanistic insight into the prebiotic effect of inulin-type fructans. Appl. Environ. Microbiol. 2009, 75, 2312-2319. [CrossRef]

10. Sonnenburg, J.L.; Chen, C.T.; Gordon, J.I. Genomic and metabolic studies of the impact of probiotics on a model gut symbiont and host. PLoS Biol. 2006, 4, e413. [CrossRef]

11. Jakobsen, L.M.; Sundekilde, U.K.; Andersen, H.J.; Nielsen, D.S.; Bertram, H.C. Lactose and Bovine Milk Oligosaccharides Synergistically Stimulate B. longum subsp. longum Growth in a Simplified Model of the Infant Gut Microbiome. J. Proteome Res. 2019, 18, 3086-3098. [CrossRef] [PubMed]

12. Aguilar-Toalá, J.; Garcia-Varela, R.; Garcia, H.; Mata-Haro, V.; González-Córdova, A.; Vallejo-Cordoba, B.; Hernández-Mendoza, A. Postbiotics: An evolving term within the functional foods field. Trends Food Sci. Technol. 2018, 75, 105-114. [CrossRef]

13. Karav, S.; Le Parc, A.; de Moura Bell, J.M.L.N.; Frese, S.A.; Kirmiz, N.; Block, D.E.; Barile, D.; Mills, D.A. Oligosaccharides released from milk glycoproteins are selective growth substrates for infant-associated bifidobacteria. Appl. Environ. Microbiol. 2016, 3622-3630. [CrossRef]

14. Sela, D.A.; Mills, D.A. Nursing our microbiota: Molecular linkages between bifidobacteria and milk oligosaccharides. Trends Microbiol. 2010, 18, 298-307. [CrossRef] [PubMed]

15. Lawley, B.; Munro, K.; Hughes, A.; Hodgkinson, A.J.; Prosser, C.G.; Lowry, D.; Zhou, S.J.; Makrides, M.; Gibson, R.A.; Lay, C.; et al. Differentiation of Bifidobacterium longum subspecies longum and infantis by quantitative PCR using functional gene targets. PeerJ 2017, 5, e3375. [CrossRef] [PubMed]

16. Garrido, D.; Ruiz-Moyano, S.; Kirmiz, N.; Davis, J.C.; Totten, S.M.; Lemay, D.G.; Ugalde, J.A.; German, J.B.; Lebrilla, C.B.; Mills, D.A. A novel gene cluster allows preferential utilization of fucosylated milk oligosaccharides in Bifidobacterium longum subsp. longum SC596. Sci. Rep. 2016, 6, 35045. [CrossRef] [PubMed]

17. Tao, N.; DePeters, E.; Freeman, S.; German, J.; Grimm, R.; Lebrilla, C.B. Bovine milk glycome. J. Dairy Sci. 2008, 91, 3768-3778. [CrossRef]

18. Brigham, C.; Caughlan, R.; Gallegos, R.; Dallas, M.B.; Godoy, V.G.; Malamy, M.H. Sialic acid (N-acetyl neuraminic acid) utilization by Bacteroides fragilis requires a novel $\mathrm{N}$-acetyl mannosamine epimerase. J. Bacteriol. 2009, 191, 3629-3638. [CrossRef]

19. Parche, S.; Beleut, M.; Rezzonico, E.; Jacobs, D.; Arigoni, F.; Titgemeyer, F.; Jankovic, I. Lactose-over-glucose preference in Bifidobacterium longum NCC2705: glcP, encoding a glucose transporter, is subject to lactose repression. J. Bacteriol. Res. 2006, 188, 1260-1265. [CrossRef]

20. Severi, E.; Hood, D.W.; Thomas, G.H. Sialic acid utilization by bacterial pathogens. Microbiology 2007, 153, 2817-2822. [CrossRef]

21. Vimr, E.R.; Kalivoda, K.A.; Deszo, E.L.; Steenbergen, S.M. Diversity of microbial sialic acid metabolism. Microbiol. Mol. Biol. Rev. 2004, 68, 132-153. [CrossRef] [PubMed]

22. Shimizu, T.; Ohtani, K.; Hirakawa, H.; Ohshima, K.; Yamashita, A.; Shiba, T.; Ogasawara, N.; Hattori, M.; Kuhara, S.; Hayashi, H. Complete genome sequence of Clostridium perfringens, an anaerobic flesh-eater. Proc. Natl. Acad. Sci. USA 2002, 99, 996-1001. [CrossRef]

23. Li, J.; Uzal, F.A.; McClane, B.A. Clostridium perfringens sialidases: Potential contributors to intestinal pathogenesis and therapeutic targets. Toxins 2016, 8, 341. [CrossRef] [PubMed]

24. Li, J.; McClane, B.A. The sialidases of Clostridium perfringens type D strain CN3718 differ in their properties and sensitivities to inhibitors. Appl. Environ. Microbiol. 2014, 80, 1701-1709. [CrossRef] 
25. Roggentin, P.; Kleineidam, R.G.; Schauer, R. Diversity in the properties of two sialidase isoenzymes produced by Clostridium perfringens spp. Biol. Chem. Hoppe-Seyler 1995, 376, 569-575. [PubMed]

26. Stewart, D.B.; Hegarty, J.P. Correlation between virulence gene expression and proton pump inhibitors and ambient $\mathrm{pH}$ in Clostridium difficile: Results of an in vitro study. J. Med. Microbiol. 2013, 62, 1517-1523. [CrossRef] [PubMed]

27. Sievers, M.; Lanini, C.; Weber, A.; Schuler-Schmid, U.; Teuber, M. Microbiology and fermentation balance in a kombucha beverage obtained from a tea fungus fermentation. Syst. Appl. Microbiol. 1995, 18, 590-594. [CrossRef]

(C) 2020 by the authors. Licensee MDPI, Basel, Switzerland. This article is an open access article distributed under the terms and conditions of the Creative Commons Attribution (CC BY) license (http://creativecommons.org/licenses/by/4.0/). 